\title{
Prognostic factors for clinical failure of exacerbations in elderly outpatients with moderate-to-severe COPD
}

This article was published in the following Dove Press journal:

International Journal of COPD

2 June 2015

Number of times this article has been viewed

\section{Robert Wilson' \\ Antonio Anzueto ${ }^{2}$ \\ Marc Miravitlles ${ }^{3}$ \\ Pierre Arvis ${ }^{4}$ \\ Daniel Haverstock ${ }^{5}$ \\ Mila Trajanovic ${ }^{6}$ \\ Sanjay Sethi ${ }^{7}$}

'Host Defence Unit, Royal Brompton Hospital, London, UK; ${ }^{2}$ University of Texas Health Science Center, South Texas Veterans Health Care System, San Antonio, TX, USA; ${ }^{3}$ Pneumology Department, Hospital Universitari Vall d'Hebron, CIBER de Enfermedades Respiratorias (CIBERES), Barcelona, Spain; ${ }^{4}$ Bayer HealthCare Pharmaceuticals, Loos, France; ${ }^{5}$ Bayer HealthCare Pharmaceuticals, Whippany, NJ, USA; ${ }^{6}$ Bayer HealthCare Pharmaceuticals, Toronto, ON, Canada; ${ }^{7}$ University at Buffalo, Buffalo, NY, USA
Correspondence: Robert Wilson Host Defence Unit, Royal Brompton Hospital, Sydney Street, London SW3 6NP, UK

Tel +442073518337

Fax +44 2073518338

Email r.wilson@rbht.nhs.uk
Background: Acute exacerbations represent a significant burden for patients with moderate-tosevere chronic obstructive pulmonary disease. Each exacerbation episode is frequently associated with a lengthy recovery and impaired quality of life. Prognostic factors for outpatients that may predict poor outcome after treatment with antibiotics recommended in the guidelines, are not fully understood. We aimed to identify pretherapy factors predictive of clinical failure in elderly ( $\geq 60$ years old) outpatients with acute Anthonisen type 1 exacerbations.

Trial registration: NCT00656747.

Methods: Based on the moxifloxacin in AECOPDs (acute exacerbations of chronic obstructive pulmonary disease) trial (MAESTRAL) database, this study evaluated pretherapy demographic, clinical, sputum bacteriological factors using multivariate logistic regression analysis, with internal validation by bootstrap replicates, to investigate their possible association with clinical failure at end of therapy (EOT) and 8 weeks posttherapy.

Results: The analyses found that the independent factors predicting clinical failure at EOT were more frequent exacerbations, increased respiratory rate and lower body temperature at exacerbation, treatment with long-acting anticholinergic drugs, and in vitro bacterial resistance to study drug. The independent factors predicting poor outcome at 8 weeks posttherapy included wheezing at preexacerbation, mild or moderate (vs extreme) sleep disturbances, lower body temperature at exacerbation, forced expiratory volume in 1 second $<30 \%$, lower body mass index, concomitant systemic corticosteroids for the current exacerbation, maintenance long-acting $\beta_{2}$-agonist and long-acting anticholinergic treatments, and positive sputum culture at EOT.

Conclusion: Several bacteriological, historical, treatment-related factors were identified as predictors of early (EOT) and later ( 8 weeks posttherapy) clinical failure in this older outpatient population with moderate-to-severe chronic obstructive pulmonary disease. These patients should be closely monitored and sputum cultures considered before and after treatment.

Keywords: AECOPD, clinical failure, prognostic factor, long-term outcome, poor outcome

\section{Introduction}

Acute exacerbations of chronic obstructive pulmonary disease (AECOPD) are the most common causes of hospitalization and death among severe COPD (chronic obstructive pulmonary disease) patients, with frequent exacerbations leading to a more rapid decline in lung function and prolonged time to recovery to previous health status as well as reducing 5-year survival. ${ }^{1-5}$ To improve survival and reduce costs, clinicians must be able to identify individuals at the highest risk for poor outcomes so that effective interventions can be implemented. 
Exacerbations become more frequent and increasingly severe as COPD progresses, with past history of frequent exacerbations increasing the likelihood of subsequent exacerbations. ${ }^{6,7}$ However, some patients are more susceptible to exacerbations than others and show worse health status and faster disease progression than those with infrequent exacerbations. ${ }^{8,9}$ The latest Global Initiative for Chronic Obstructive Lung Disease document recommends antibiotics and systemic corticosteroids for the management of AECOPD. ${ }^{4}$ Inadequate or insufficient antimicrobial therapy may be responsible for exacerbation recurrence; however, historical patient characteristics and disease severity may be equally as important as exacerbation severity regarding its symptoms. In particular, older age ( $>65$ years), presence of severe COPD (forced expiratory volume in 1 second $\left[\mathrm{FEV}_{1}\right]<50 \%$ ), frequent exacerbations ( $\geq 3$ in the previous 12 months), and comorbid cardiac disease have previously been identified as predictors of a higher risk of poor outcomes and also identify patients as having complicated COPD. ${ }^{10-14}$ Stratifying patients according to these host factors can help the management of patients at high risk for unfavorable outcomes, with guidelines recommending adequate, appropriate antibiotic treatment for these individuals, amoxicillin/clavulanic acid (AMC) being most often listed and a respiratory fluoroquinolone is also commonly included..$^{11,15,16}$ Nevertheless, although these factors may help guide management, more information is needed on the mechanisms of failure and rapid relapse, particularly in high risk individuals.

The MAESTRAL (Moxifloxacin in AECOPDs trial) study enrolled 1,352 elderly outpatients with Anthonisen type 1 exacerbations and moderate-to-severe $\mathrm{COPD}\left(\mathrm{FEV}_{1}<60 \%\right.$ predicted) and compared the clinical efficacy of moxifloxacin (MXF) and AMC with regards to clinical failure rate at 8 weeks posttherapy. ${ }^{17}$ The study showed that MXF was noninferior to AMC, with MXF also significantly reducing the clinical failure rate vs AMC, in patients with microbiologically confirmed bacterial exacerbations. ${ }^{17}$

The current post hoc analyses of the MAESTRAL cohort sought to determine whether new factors indicative of poor outcome of AECOPD could be identified in this population when AECOPD is treated with antibiotics \pm systemic corticosteroids consistent with current guidelines. ${ }^{18}$

\section{Materials and methods Study design}

MAESTRAL was a Phase III, prospective, multinational, randomized, double-blind, double-dummy, noninferiority, controlled study, comparing the efficacy and safety of 5-day oral MXF $400 \mathrm{mg}$, once daily with 7-day oral AMC $875 / 125 \mathrm{mg}$, twice daily in elderly ( $\geq 60$ years old) outpatients with a history of AECOPD with an Anthonisen type 1 exacerbation (NCT00656747). ${ }^{17,19}$ Randomization was stratified based on concomitant administration of a 5-day course of systemic steroids $(30-40 \mathrm{mg} / \mathrm{d}$ prednisolone or equivalent, according to physician preference) for the current exacerbation at the treating physician's discretion to ensure equal numbers of steroid treated patients per arm. The study protocol was approved by the ethics committees, and patients provided their written informed consent.

Spontaneous sputum samples were obtained from all patients and assessed in a local laboratory by Gram stain, culture, and susceptibility testing at enrollment, during therapy, at end of therapy (EOT), and at 4 and 8 weeks posttherapy. Sputum samples were sent to a central laboratory where reidentification of organisms and culture positivity was confirmed. Further details of the study design and the primary results have been reported previously. ${ }^{17,20}$

The primary efficacy endpoint was a clinical failure at 8 weeks posttherapy in the per-protocol population, and the study was powered to show noninferiority between treatments. ${ }^{17}$ Clinical failure was defined as the requirement for additional or alternate treatment for an exacerbation of respiratory symptoms, with systemic antibiotics and/or systemic corticosteroids (including increased dose or duration of treatment) and/or hospitalization with systemic antibiotic and/or systemic corticosteroid administration within 8 weeks posttherapy.

Post hoc analyses presented in this paper were conducted in the intent-to-treat (ITT) population. Clinical factors analyzed were various demographic parameters (including geographical location), age, employment status, medical history, Acute Exacerbation of Chronic Bronchitis Symptom Scale factors, ${ }^{21}$ and aspects of exacerbation history. The influence of medications was also investigated, including coadministration of systemic corticosteroids for the current exacerbation or maintenance therapy with inhaled corticosteroids, bronchodilators (eg, xanthine derivatives, short- and long-acting $\beta_{2}$-agonists [SABA and LABA], and short- and long-acting anticholinergics [SAMA and LAMA]). Microbiological factors investigated included presence and number of species in the sputum samples collected during the study (ie, pretherapy, during therapy, EOT, 4 and 8 weeks posttherapy) and in vitro resistance of isolated organisms to study drugs.

\section{Statistical analyses}

The association with clinical failure at EOT and at 8 weeks posttherapy of 59 variables described briefly earlier was 
evaluated by univariate and multivariate stepwise logistic regression analyses. The threshold for significance was set at $P<0.1$ for variables in the univariate model (data not shown). The independent prognostic contributions of these variables were then entered into the multivariate logistic regression model to determine which factors remained significantly predictive in the presence of the others, using a threshold for significance of $P<0.05$. In the first step, a stepwise (backward eliminating) logistic regression analysis was performed to identify the independent predictors. Second, the identified parameters were included in a one-step logistic regression model to calculate odds ratios and $95 \%$ confidence intervals presented in this manuscript. Treatment (MXF vs AMC) was included in the logistic regression model regardless of its significance level. ${ }^{22}$ The goodness-of-fit of the model $\left(\chi^{2}\right)$ was evaluated by Hosmer and Lemeshow test with $P>0.05$ as threshold, with higher $P$-value indicating better fit. To estimate the performance and efficiency of the logistic regression model (or to check overfitting), bootstrap approach was implemented with 500 and 1,000 randomly selected replicates from the entire dataset. Discrimination of the model was determined by receiver operating characteristics curve analysis, and the area under the curve was determined. Variables were considered as either continuous or categorical. Parameters of individual patients were not analyzed if values were missing.

\section{Results}

The ITT population consisted of 1,352 randomized patients with the majority being $\geq 65$ years $(72 \%)$, being male $(80 \%)$, having at least one coexisting illness $(80 \%)$, 35\% had systemic corticosteroid treatment for the current exacerbation, and on average patients had (mean \pm standard deviation) $2.5 \pm 1.1$ exacerbations in the past 12 months (Table 1). Clinical failure rate in the total ITT population was $7.6 \%$ at the EOT and $21.0 \%$ at 8 weeks posttherapy.

Univariate analyses identified several variables that were related to clinical outcome at both EOT and 8 weeks posttherapy (data not shown). The multivariate analysis revealed a number of predictive factors significantly associated with clinical failure either at EOT (Figure 1) and/or 8 weeks posttherapy (Figure 2).

\section{End of therapy}

Prognostic factors associated with a higher risk of clinical failure at EOT included increased frequency of exacerbations in the previous 12 months, clinical assessments of lower body temperature (BT) and increased respiratory rate at the current exacerbation, maintenance treatment with LAMA, and in vitro bacterial resistance to study drug pretherapy (Figure 1).

\section{8 weeks posttherapy}

Clinical failures at EOT were carried forward to 8 weeks posttherapy; this includes all true failures and relapses. At 8 weeks posttherapy, lower BT continued to be highly predictive for clinical failure in the multivariate analysis. Wheezing at preexacerbation was associated with increased risk of clinical failure, whereas patients with severe sleep disturbances had less failures. Additional significant predictors included markers of disease severity such as lower body mass index (BMI) and lower $\mathrm{FEV}_{1}(<30 \%$ predicted) (Figure 2).

Patients' treatments were associated with their risk of failure. Patients receiving systemic corticosteroids (but not those receiving inhaled medication) for the current exacerbation, maintenance LABA and LAMA treatments were at higher risk of clinical failure. We looked at whether patients on systemic corticosteroids, LABA, or LAMA therapy had additional characteristics for being at higher risk of poor outcome.

Patients who received systemic corticosteroids for their exacerbation (vs those who did not) had a history of respiratory disorders, $\mathrm{FEV}_{1}<30 \%$ (predicted), greatly increased dyspnea, increased heart rate ( $>90$ beats/min) and respiratory rate $(>20 / \mathrm{min})$ more frequently, and reported cough very frequently/all the time. ${ }^{17}$

Patients with maintenance LABA therapy (vs those without) had more comorbidities (eg, diabetes [14.6\% vs $9.4 \%$ of patients, $P<0.01$ ], history of cardiac disorders [26.9\% vs $16.6 \%$ of patients, $P<0.01$ ], cardiopulmonary disease $[21.1 \%$ vs $8.6 \%$ of patients, $P<0.01$ ], history of respiratory failure [19.5\% vs $8.0 \%$ of patients, $P<0.01]$ ), more exacerbations in the previous 3 months ( $52.9 \%$ vs $46.6 \%$ of patients, $P<0.05$ ), and raised BT ( $34.8 \%$ vs $21.4 \%$ of patients, $P<0.01$ ), reflecting more severe disease status, respectively.

Patients who received LAMA therapy were more frequently treated with systemic corticosteroids for the current exacerbation $(40.4 \%$ vs $32.8 \%, P<0.01)$ and short-acting bronchodilators $(29.8 \%$ vs $23.2 \%, P<0.01)$, had greater history of respiratory failure $(16.6 \%$ vs $8.1 \%, P<0.01)$ and lower BT $\left(\mathrm{eg}, \leq 36^{\circ} \mathrm{C} ; 13.6 \%\right.$ vs $\left.8.0 \%, P<0.01\right)$ compared with those without LAMA treatment, respectively. LAMAtreated patients were mainly enrolled in Europe/Canada/ South Africa.

Of the microbiological results investigated, only bacteriologically positive sputum culture at EOT was found to be 
Table I Baseline demographic and disease characteristics of randomized patients in the ITT $(\mathrm{N}=$ I,352) population

\begin{tabular}{|c|c|c|c|}
\hline Characteristic & $\begin{array}{l}\text { Moxifloxacin } \\
(\mathrm{N}=677)\end{array}$ & $\begin{array}{l}\text { Amoxicillin/clavulanic } \\
\text { acid }(N=675)\end{array}$ & $\begin{array}{l}\text { Total } \\
(\mathrm{N}=1,352)\end{array}$ \\
\hline \multicolumn{4}{|l|}{ Geographic region, $n(\%)$} \\
\hline Asia Pacific & $205(30)$ & $212(3 \mid)$ & $4 \mid 7(3 \mid)$ \\
\hline Europe & $249(37)$ & $257(38)$ & $506(37)$ \\
\hline South Africa & $27(4)$ & $15(2)$ & $42(3)$ \\
\hline Latin America & $181(27)$ & $181(27)$ & $362(27)$ \\
\hline Canada & $15(2)$ & $10(1)$ & $25(2)$ \\
\hline Male sex, n (\%) & $534(79)$ & $545(8 \mathrm{I})$ & $\mathrm{I}, 079(80)$ \\
\hline \multicolumn{4}{|l|}{ Race, n (\%) } \\
\hline Caucasian & $4 \mid 3(6 \mid)$ & $403(60)$ & $816(60)$ \\
\hline Asian & $209(31)$ & $213(32)$ & $422(31)$ \\
\hline Other & $55(8)$ & $59(9)$ & $114(8)$ \\
\hline Age (years), mean $\pm S D$ & $69.6 \pm 6.8$ & $69.6 \pm 6.6$ & $69.6 \pm 6.7$ \\
\hline Range & 59-93 & $60-91$ & 59-93 \\
\hline$\geq 65$ years, $n(\%)$ & $486(72)$ & $492(73)$ & $978(72)$ \\
\hline $\mathrm{BMI}\left(\mathrm{kg} / \mathrm{m}^{2}\right)$, mean $\pm \mathrm{SD}$ & $25.0 \pm 5.4$ & $24.7 \pm 5.2$ & $24.8 \pm 5.3$ \\
\hline Current smokers, n (\%) & $149(22)$ & |44 (2I) & $293(22)$ \\
\hline Coexisting illnesses, n (\%) & $533(79)$ & $545(8 I)$ & $\mathrm{I}, 078(80)$ \\
\hline Coronary artery disease & $38(6)$ & $27(4)$ & $65(5)$ \\
\hline Congestive heart failure & $34(5)$ & $42(6)$ & $76(6)$ \\
\hline Peripheral artery disease & $15(2)$ & $5(I)$ & $20(I)$ \\
\hline Renal dysfunction & $26(4)$ & $21(3)$ & $47(3)$ \\
\hline Liver dysfunction & $13(2)$ & $14(2)$ & $27(2)$ \\
\hline Diabetes mellitus & $67(10)$ & $77(\mathrm{II})$ & I 44 (II) \\
\hline Any respiratory comedication, $\mathrm{n}(\%)$ & $628(93)$ & $621(92)$ & $1,249(92)$ \\
\hline Short or long-acting $\beta_{2}$-agonists & $368(54)$ & $364(54)$ & $732(54)$ \\
\hline Inhaled steroids ${ }^{a}$ & $389(57)$ & $37 \mid(55)$ & $760(56)$ \\
\hline Ipratropium or tiotropium & $305(45)$ & $310(46)$ & $615(45)$ \\
\hline Xanthine derivatives & $222(33)$ & $219(32)$ & $44 \mid(33)$ \\
\hline Previous antimicrobial use, ${ }^{\mathrm{b}} \mathrm{n}(\%)$ & $235(35)$ & $227(34)$ & $462(34)$ \\
\hline Systemic steroid use, n (\%) & $236(35)$ & $239(35)$ & $475(35)$ \\
\hline \multicolumn{4}{|l|}{ Lung function at enrollment } \\
\hline $\mathrm{FEV}, \%$ predicted, mean $\pm \mathrm{SD}$ & $38.4 \pm 11.6$ & $38.7 \pm 11.7$ & $38.6 \pm 11.7$ \\
\hline $\mathrm{FEV}_{1}(\mathrm{~L})$, mean $\pm \mathrm{SD}$ & $0.982 \pm 0.370$ & $0.978 \pm 0.360$ & $0.980 \pm 0.365$ \\
\hline $\mathrm{FEV}_{1}<30 \%, \mathrm{n}(\%)$ & $174(26)$ & $165(24)$ & $339(25)$ \\
\hline \multicolumn{4}{|l|}{ Exacerbations in previous year } \\
\hline Mean \pm SD & $2.5 \pm 1.1$ & $2.5 \pm 1.1$ & $2.5 \pm 1.1$ \\
\hline Range & $1-15$ & $1-10$ & $1-15$ \\
\hline \multicolumn{4}{|l|}{$\begin{array}{l}\text { Time since last exacerbation } \\
\text { (days) }\end{array}$} \\
\hline Mean \pm SD & $109.6 \pm 65.2$ & $103.6 \pm 61.1$ & $106.6 \pm 63.2$ \\
\hline
\end{tabular}

Notes: ancludes combination therapy (steroid plus bronchodilators). ${ }^{b}$ Any antimicrobial given for any indication between 30 and 90 days prior to enrollment. Abbreviations: BMI, body mass index; FEV , forced expiratory volume in I second; ITT, intent-to-treat; SD, standard deviation.

predictive of clinical failure at 8 weeks posttherapy; other bacteriological factors such as resistance to study drugs was not associated with risk of failure at this time point.

Geographic region also showed statistical significance with patients in Asia Pacific (AP), demonstrating increased risk of clinical failure compared with Latin America (LA) as reference category (with lowest rate of clinical failure). This observation may be driven by the higher proportion of patients with $\mathrm{FEV}_{1}<30 \%$ (predicted) (AP: $34 \%$ vs LA: $26 \%$, respectively) and higher mean number of exacerbations in the previous 12 months (AP: $3.0 \pm 1.5$ vs LA: $2.2 \pm 0.5$, respectively). It is also notable that in AP, fewer patients received systemic corticosteroids (AP: $27.8 \%$ vs LA: $40.9 \%$, respectively) and had comorbidities (AP: $62.8 \%$ vs LA: $87.3 \%$, respectively).

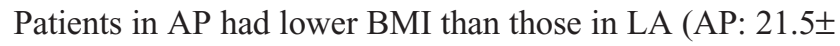
$3.7 \mathrm{~kg} / \mathrm{m}^{2}$ vs LA: $25.6 \pm 4.9 \mathrm{~kg} / \mathrm{m}^{2}$, respectively).

\section{Discrimination and goodness-of-fit analyses}

Discrimination of the models at EOT and 8 weeks posttherapy was tested by receiver operating characteristics analysis to estimate the probability of true positive failures. 


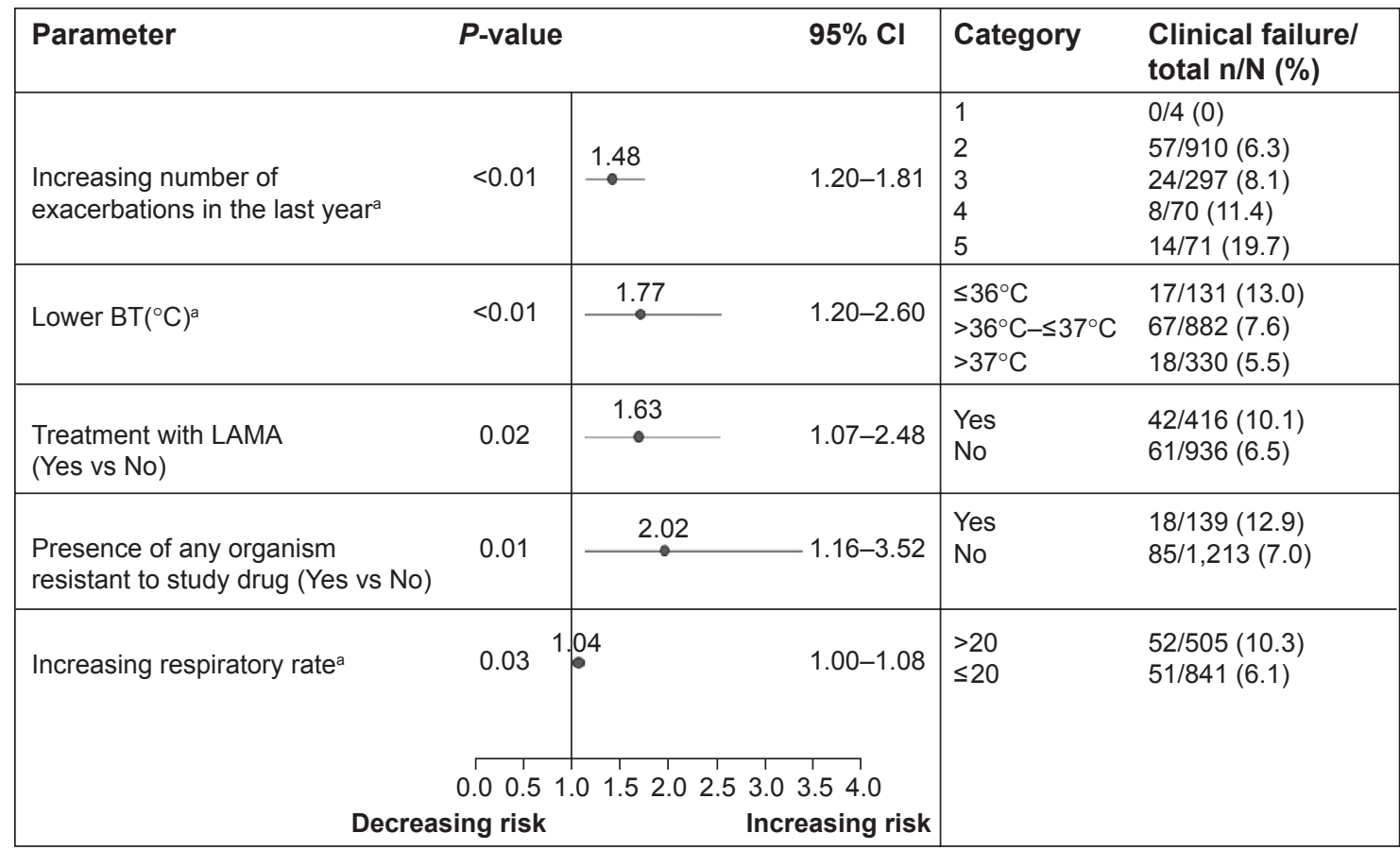

Figure I Prognostic factors associated with increased risk of clinical failure at EOT based on multivariate analysis. Notes: Odds ratio is shown with horizontal line presentation of $95 \% \mathrm{Cl}$. ${ }^{a}$ Continuous variable.

Abbreviations: BT, body temperature; $\mathrm{Cl}$, confidence interval; EOT, end of therapy; LAMA, long-acting anticholinergic; N, number of patients in category; n, number of patients with clinical failures.

The analyses provided an area under the curve of 0.6639 for the EOT logistic regression model (weaker) and of 0.7274 (relatively strong) for the 8 weeks posttherapy model.

To test the predictive strength of the logistic regression models, Hosmer and Lemeshow test was performed. The predictive strength of the EOT and 8 weeks posttherapy logistic regression models is demonstrated by the goodness-of-fit $P$-values of 0.53 and 0.98 , respectively (ie, $P>0.05$ ). Internal validation of the models was implemented in bootstrap analyses by using 500 and 1,000 random replicates selected from the entire dataset for both time points, confirming the results of the logistic regression analysis (data not shown).

\section{Discussion}

Patients in this large $(\mathrm{N}=1,352)$ study met current definitions of complicated COPD: being elderly ( $\geq 60$ years old) with moderate-to-severe airway obstruction (all with $\mathrm{FEV}_{1}<60 \%$, $25 \%$ with $\mathrm{FEV}_{1}<30 \%$ ), $\geq 2$ exacerbations per year, and $80 \%$ with comorbid conditions. ${ }^{17}$ However, as it can be seen in this analysis (Table 1), many patients did not receive guidelineapproved treatment (eg, LABA and LAMA). The post hoc analyses presented in this paper have identified several novel observations that may help predict treatment failures of AECOPD when treated according to the guidelines. Consideration of the results is also helpful, highlighting hypotheses about the complexity of the underlying pathomechanisms of acute exacerbation of COPD. Frequent exacerbations and subsequent hospitalizations worsen quality of $l i f \mathrm{e}^{23}$ and increase mortality risk; ${ }^{5}$ therefore, avoiding clinical failure in such patients has both short- and long-term benefits.

These analyses confirm the results of previous studies showing that more frequent exacerbations increase the risk of failure at EOT and more severe airflow obstruction is associated with poor outcome for up to 8 weeks posttherapy. ${ }^{10,13,24}$ However, all enrolled patients had a $\mathrm{FEV}_{1}<60 \%$ and $\geq 2$ exacerbations in the previous year, and this may account for frequent exacerbations not influencing failure at 8 weeks posttherapy and the influence of low $\mathrm{FEV}_{1}$ being less influential than in previous studies.

Interestingly, age and cardiac comorbidity were not found to be predictive of poor outcome in this study in contrast to previous studies. ${ }^{12,13}$ The lack of effect of older age is likely due to the threshold of 60 years required for inclusion and $72 \%$ of the study population being $\geq 65$ years, narrowing the age spectrum for this analysis. Similarly, over two-thirds of patients had a cardiovascular comorbidity, reflecting the elderly population and smoking history, thereby, making the influence of this variable difficult to determine. ${ }^{17}$ It should also be noted that patients with clinically relevant bradycardia, history of symptomatic arrhythmias, and heart 


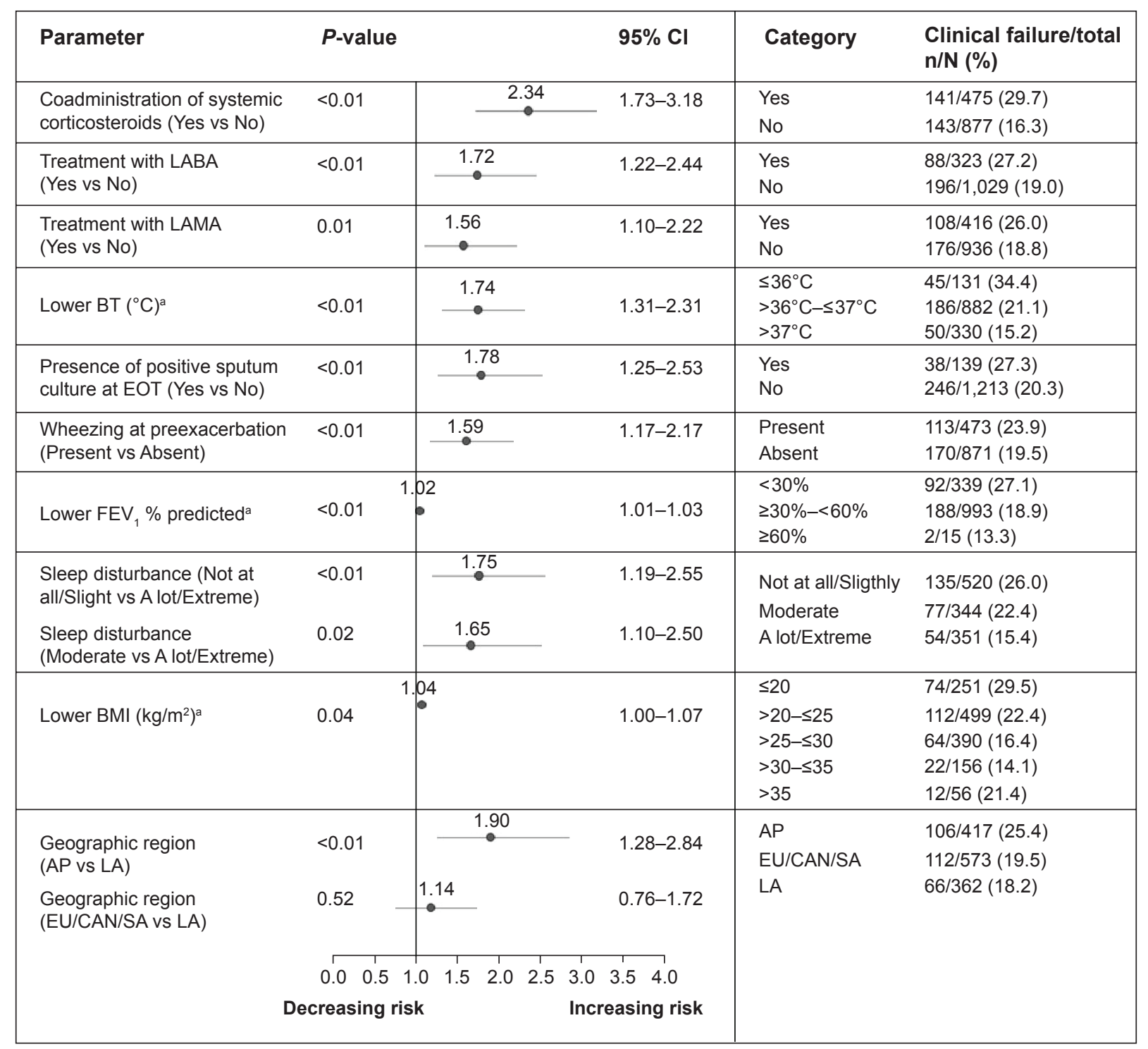

Figure 2 Prognostic factors associated with increased risk of clinical failure at 8 weeks posttherapy based on multivariate analysis.

Notes: Odds ratio is shown with horizontal line presentation of $95 \% \mathrm{Cl}$. ${ }^{\mathrm{a} C}$ Continuous variable.

Abbreviations: AP, Asia Pacific; BMI, body mass index; BT, body temperature; CAN, Canada; $\mathrm{Cl}$, confidence interval; EOT, end of therapy; EU, Europe; FEV expiratory volume in I second; LA, Latin America; LABA, long-acting $\beta_{2}$-agonist; LAMA, long-acting anticholinergic; N, number of patients in category; n, number of patients with clinical failures; SA, South Africa.

failure with documented reduced left-ventricular-ejection fraction were excluded from the study, according to the label of MXF. ${ }^{17}$

Previous antibiotic trials of AECOPD have not shown clear associations between baseline bacteriology and bacteriological response to clinical outcomes in AECOPD. An important finding in MAESTRAL was that pretherapy presence of bacterial pathogens resistant to study drugs predicted clinical failure at EOT, and the presence of a positive bacterial culture at EOT increased the risk of clinical relapse over subsequent weeks. Sputum cultures are currently not advocated in the routine management of AECOPD. ${ }^{4}$ Our data suggest that pathogen-directed treatment (particularly when at risk of carrying resistant pathogens) could be beneficial for outpatients and may prevent clinical failure and relapses after treatment. Therefore, sputum cultures should be considered in at-risk patients.

Among the novel factors identified with poor outcome, the most surprising observation was that the use of oral corticosteroids at exacerbation was associated with worse clinical outcomes at 8 weeks but not failure at the end of the treatment. The obvious explanation is that treating physicians determined the use of oral corticosteroids in patients who had more severe disease; ${ }^{17}$ a worse outcome in those individuals 
could, therefore, be expected. Among corticosteroid-treated patients, a significantly larger proportion had a history of respiratory disorders, $\mathrm{FEV}_{1}<30 \%$ predicted, greatly increased dyspnea, increased heart rate and respiratory rate, and very frequent cough at the "current exacerbation"; thus presenting with more severe exacerbations. ${ }^{17}$ Previous studies have demonstrated that oral corticosteroids reduce treatment failures in exacerbations in hospitalized patients and those attending emergency departments. None of these studies examined an outpatient office-based population as was conducted in the MAESTRAL study. ${ }^{25-30}$ An important difference between these previous trials and the current study is that the antibiotics used here were appropriate for the severity of these patients, while in some previous studies, antibiotics of limited bacteriological efficacy were used ${ }^{29,31}$ or were not documented. ${ }^{26,30}$ Another significant difference is that while previous studies included all Anthonisen grades of nonhospitalized patients, this study included only type 1 exacerbations in which the likelihood of bacterial infection was greater.

Not only those patients receiving systemic corticosteroids during exacerbation, but also those receiving LABA and/or LAMA long-term were more likely to relapse during the following 8 weeks. Our analyses suggest that this is most likely because patients had more severe diseases and more comorbidities as described above. However, despite these treatments, patients remain at higher risk of failure; therefore, alternative management needs to be explored. Targeted antibiotic treatment guided by sputum cultures at exacerbation and EOT may be one such approach. Among these three treatments, LAMA treatment was the only one associated with early failure at EOT. Wheezing at preexacerbation was another factor associated with failure at 8 weeks posttherapy, which may be reflective of disease severity, making relapses more likely to occur.

A novel observation in this study was that the presence of lower BT (ie, $\leq 36^{\circ} \mathrm{C}$ ) during exacerbation increased the risk of clinical failure at EOT and 8 weeks posttherapy. The body's thermogenic capacity, in both healthy and infected individuals, is centrally regulated and depends on nutritional status, basal metabolic rate, diet, and physical activity. ${ }^{32,33}$ Lower BT may be the result of poor health status and malnutrition (represented as BMI $<20 \mathrm{~kg} / \mathrm{m}^{2}$ and/or decreased fat-free mass) that are frequently observed in COPD patients. This analysis also showed that patients with the lowest BMI values were at the highest risk of experiencing clinical failure. COPD patients often suffer from low appetite, weight loss, fatigue, muscle dysfunction, and other metabolic impairments, which likely influence their respiratory function and physical activity level. ${ }^{34}$ The effect of low BMI may have been marginally significant in the analysis because of an increased clinical failure rate at 8 weeks posttherapy in patients with a BMI of $>35 \mathrm{~kg} / \mathrm{m}^{2}$. Previously, hypothermia has been known to predict worse outcomes in critically ill patients, ${ }^{35,36}$ particularly the elderly, ${ }^{35,36}$ and it may increase the risk of pneumonia ${ }^{37}$ or mortality ${ }^{36}$ and could be indicative of an inadequate host response to infection. ${ }^{33}$ In our study, patients who had higher BT at exacerbation (ie, $>37^{\circ} \mathrm{C}$ ) showed the lowest risk of clinical failure at EOT and 8 weeks posttherapy. Of note, patients with $\mathrm{BT} \leq 36^{\circ} \mathrm{C}$ were more likely to be receiving LAMA treatment $(13.6 \%$ vs $8.0 \%$, respectively; $P<0.01)$.

Increased respiratory rate, a parameter reflecting the severity of the acute exacerbation episode, was associated with worse outcomes at EOT. Sleep disturbances are common in COPD patients (up to $75 \%$ of patients) and could be linked to increased exacerbation frequency and impaired quality of life. ${ }^{38}$ However, in the present analysis, the presence of more severe sleep disturbances during the presenting exacerbation episode was associated with lower likelihood of relapse in the following 8 weeks. This observation seems counterintuitive, but these patients might have received more supporting therapy. ${ }^{17}$

Our results present a number of predictive variables (at EOT: 5 variables, at 8 weeks posttherapy: 10 variables) which were identified in a step-by-step statistical analysis and, furthermore, validated by bootstrapping and goodnessof-fit tests, providing a rather strong prediction model for such patients, particularly for the follow-up period. However, these results should be generalized with great caution to the general COPD population and best applied to those elderly with complicated COPD, experiencing an Anthonisen type 1 exacerbation. $^{22}$

In conclusion, this study has identified several clinically useful predictive factors for clinical failure in AECOPD, in addition to known factors such as age, severity of airflow obstruction, frequency of previous exacerbations, and cardiac comorbidity. Closer attention should be paid to the frequency of exacerbations and severity of underlying COPD, as gradations observed in these parameters influence clinical outcome. Severity of changes in wheezing at preexacerbation, respiratory rate, and BT at exacerbation should be warning signals of poorer outcome. In addition, markers of disease severity such as low BMI, use of systemic corticosteroids, and LAMA and LABA treatments can be used to identify patients at risk for relapse following 
treatment recommended in the guidelines. Our findings also emphasize the need for further studies investigating important questions, particularly whether evaluation of sputum microbiology and pathogen-directed therapy should be recommended to minimize the risk of poor outcomes. Sputum culture is currently not recommended for outpatients with AECOPD. However, the association of the presence of bacterial pathogens resistant to study drugs and positive bacterial culture at EOT with worse clinical outcomes strongly suggests that this question should be revisited. As new therapies are developed to treat infection and inflammation in AECOPD, enrollment of patients with previously known and newly identified poor prognostic factors will need to be identified to ensure that studies are adequately powered to include well-balanced study groups and to allow appropriate subgroup analyses. In the interim, patients at risk of poorer outcomes should be closely monitored before, during, and after treatment of their exacerbation and additional interventions implemented to resolve the exacerbation and prevent relapses.

\section{Acknowledgments}

This study was supported by Bayer HealthCare, Germany. Editorial assistance was provided by Highfield Communication, Oxford, UK, sponsored by Bayer HealthCare. The authors thank Mr Feng Zhan, inVentive Health, US, for his invaluable support in the bootstrapping and logistic regression analyses.

\section{Author contributions}

Robert Wilson, Antonio Anzueto, Marc Miravitlles, Pierre Arvis, and Sanjay Sethi contributed to the study design, data analysis and interpretation, and review and approval of the manuscript preparation. Robert Wilson is the guarantor of this publication. Daniel Haverstock and Mila Trajanovic contributed to statistical analysis and interpretation, and data analysis and interpretation, respectively, and contributed to review and approval of the manuscript preparation. All authors contributed toward data analysis, drafting and revising the paper and agree to be accountable for all aspects of the work.

\section{Disclosure}

Sanjay Sethi has received lecture, consulting fees, and research funds from Bayer, GlaxoSmithKline, Merck, and Sharp \& Dohme. Antonio Anzueto has participated as a speaker in scientific meetings or courses organized and financed by various pharmaceutical companies including AstraZeneca, Boehringer Ingelheim, Bayer, Pfizer, GlaxoSmithKline, and Novartis.
Antonio Anzueto has been a consultant for AstraZeneca, Boehringer Ingelheim, Pfizer, GlaxoSmithKline, Novartis, and Bayer. He has also been the principal investigator for research grants for the University of Texas Health Science Center (San Antonio, TX, USA) that received payment for participating in a multicenter clinical trial sponsored by: GlaxoSmithKline, Bayer, Eli Lilly, and National Institutes of Health. Marc Miravitlles has received speaker fees from Boehringer Ingelheim, Pfizer, AstraZeneca, Bayer Schering, Novartis, Talecris-Grifols, Takeda-Nycomed, Merck, Sharp \& Dohme, and Novartis, and consulting fees from Boehringer Ingelheim, Pfizer, GlaxoSmithKline, AstraZeneca, Bayer Schering, Novartis, Almirall, Merck, Sharp \& Dohme, Talecris-Grifols, and Takeda-Nycomed. Robert Wilson has received honoraria for taking part in advisory boards and presenting at meetings from Almirall, Aperion Advisors LLC, AstraZeneca, Athena Medical PR, Bayer HealthCare, Forest Laboratories (Bronchiectasis symposium), Genactis Ltd, Opticom International, Penn Technology Partnership, Resolutions Group, Rivervest, Transave, VacZine Analytics, and Wyeth Pharmaceuticals. Pierre Arvis is a full-time employee of Bayer HealthCare. Daniel Haverstock is a full-time employee of Bayer HealthCare Pharmaceuticals. Mila Trajanovic is a full-time employee of Bayer Inc. and holds stocks of the company. The authors report no other conflicts of interest in this work.

\section{References}

1. Donaldson GC, Seemungal TA, Bhowmik A, et al. Relationship between exacerbation frequency and lung function decline in chronic obstructive pulmonary disease. Thorax. 2002;57(10):847-852.

2. Spencer S, Jones PW; GLOBE Study Group. Time course of recovery of health status following an infective exacerbation of chronic bronchitis. Thorax. 2003;58:589-593.

3. Sialer S, Adamantia L, Guerrero M, et al. Relation between chronic obstructive pulmonary disease and antibiotics. Curr Infect Dis Rep. 2012; 14:300-307.

4. Vestbo J, Hurd SS, Agustí AG, et al. Global strategy for the diagnosis, management, and prevention of chronic obstructive pulmonary disease: GOLD executive summary. Am J Respir Crit Care Med. 2013;187(4):347-365.

5. Soler-Cataluna JJ, Martinez-Garcia MA, Roman Sanchez P, et al. Severe acute exacerbations and mortality in patients with chronic obstructive pulmonary disease. Thorax. 2005;60:925-931.

6. Ball P, Harris JM, Lowson D, et al. Acute infective exacerbations of chronic bronchitis. QJM. 1995;88(1):61-68.

7. Dever LL, Shashikumar K, Johanson WG. Antibiotics in the treatment of acute exacerbations of chronic bronchitis. Expert Opin Investig Drugs. 2002;11:911-925.

8. Wedzicha JA, Seemungal TA. COPD exacerbations: defining their cause and prevention. Lancet. 2007;370:786-796.

9. Hurst JR, Vestbo J, Anzueto A, et al. Susceptibility to exacerbation in chronic obstructive pulmonary disease. $N$ Engl J Med. 2010;363(12): $1128-1138$.

10. Miravitlles M, Guerrero T, Mayordomo C, et al. Factors associated with increased risk of exacerbation and hospital admission in a cohort of ambulatory COPD patients: a multiple logistic regression analysis. The EOLO Study Group. Respiration. 2000;67(5):495-501. 
11. Murphy TF, Sethi S. Chronic obstructive pulmonary disease. Role of bacteria and guide to antibacterial selection in the older patient. Drugs Aging. 2002;19:761-775.

12. Wilson R, Allegra L, Huchon G, et al. Short and long-term outcomes of moxifloxacin compared to standard antibiotic treatment in acute exacerbations of chronic bronchitis. Chest. 2004;125:953-964.

13. Wilson R, Jones P, Schaberg T, et al; MOSAIC Study Group. Antibiotic treatment and factors influencing short and long term outcomes of acute exacerbations of chronic bronchitis. Thorax. 2006;61:337-342.

14. Anzueto A, Miravitlles M, Ewig S, et al. Identifying patients at risk of late recovery ( $>8$ days) from acute exacerbation of chronic bronchitis and COPD. Respir Med. 2012;106:1258-1267.

15. O'Donnell DE, Hernandez P, Kaplan A, et al. Canadian Thoracic Society recommendations for management of chronic obstructive pulmonary disease - 2008 update - highlights for primary care. Can Respir J. 2008;15(Suppl A):1A-8A.

16. Sethi S, Murphy TF. Infection in the pathogenesis and course of chronic obstructive pulmonary disease. $N$ Engl J Med. 2008;359:2355-2365.

17. Wilson R, Anzueto A, Miravitlles M, et al. Moxifloxacin vs amoxicillin/ clavulanic acid in outpatient AECOPD: MAESTRAL results. Eur Respir J. 2012;40(1):17-27.

18. Woodhead M, Blasi F, Ewig S, et al. Guidelines for the management of adult lower respiratory tract infections - full version. Clin Mirobiol Infect. 2011;17(Suppl 6):E1-E59.

19. Anthonisen NR, Manfreda J, Warren CP, et al. Antibiotic therapy in exacerbations of chronic obstructive pulmonary disease. Ann Intern Med. 1987;106:196-204.

20. Wilson R, Anzueto A, Miravitlles M, et al. A novel study design for antibiotic trials in acute exacerbations of COPD: MAESTRAL methodology. Int J Chron Obstruct Pulmon Dis. 2011;6:373-383.

21. Jones PW. The Acute Exacerbation of COPD Symptom Scale (AECB-SS). J COPD Management. 2006; Quarter 2, article 3.

22. Labarere J, Renaud B, Fine MJ. How to derive and validate clinical prediction models for use in intensive care medicine. Intensive Care Med. 2014;40(4):513-527.

23. Seemungal TA, Donaldson GC, Paul EA, et al. Effect of exacerbation on quality of life in patients with chronic obstructive pulmonary disease. Am J Respir Crit Care Med. 1998;157(5 Pt 1):1418-1422.

24. Niewoehner DE, Collins D, Erbland ML. Relation of FEV1 to clinical outcomes during exacerbations of chronic obstructive pulmonary disease. Department of Veterans Affairs Cooperative Study Group. Am J Respir Crit Care Med. 2000;161(4 Pt 1):1201-1205.

25. Thompson WH, Nielson CP, Carvalho P, et al. Controlled trial of oral prednisone in outpatients with acute COPD exacerbation. Am J Respir Crit Care Med. 1996;154(2 Pt 1):407-412.
26. Davies L, Angus RM, Calverley PM. Oral corticosteroids in patients admitted to hospital with exacerbations of chronic obstructive pulmonary disease: a prospective randomised controlled trial. Lancet. 1999;354(9177):456-460.

27. Niewoehner DE, Erbland ML, Deupree RH, et al. Effect of systemic glucocorticoids on exacerbations of chronic obstructive pulmonary disease. Department of Veterans Affairs Cooperative Study Group. N Engl J Med. 1999;340(25):1941-1947.

28. Maltais F, Ostinelli J, Bourbeau J, et al. Comparison of nebulized budesonide and oral prednisolone with placebo in the treatment of acute exacerbations of chronic obstructive pulmonary disease: a randomized controlled trial. Am J Respir Crit Care Med. 2002;165(5): 698-703.

29. Aaron SD, Vandemheen KL, Hebert P, et al. Outpatient oral prednisone after emergency treatment of chronic obstructive pulmonary disease. N Engl J Med. 2003;348(26):2618-2625.

30. $\mathrm{Li} \mathrm{H}, \mathrm{He} \mathrm{G}, \mathrm{Chu} \mathrm{H}$, et al. A step-wise application of methylprednisolone versus dexamethasone in the treatment of acute exacerbations of COPD. Respirology. 2003;8(2):199-204.

31. Daniels JM, Snijders D, de Graaff CS, et al. Antibiotics in addition to systemic corticosteroids for acute exacerbations of chronic obstructive pulmonary disease. Am J Respir Crit Care Med. 2010;181:150-157.

32. Nakamura K. Central circuitries for body temperature regulation and fever. Am J Physiol Regul Integr Comp Physiol. 2011;301: R1207-R1228.

33. Van Marken LWD, Schrauwen P. Implications of nonshivering thermogenesis for energy balance regulation in humans. Am J Physiol Regul Integr Comp Physiol. 2011;301:R285-R296.

34. Raguso CA, Luthy C. Nutritional status in chronic obstructive pulmonary disease: role of hypoxia. Nutrition. 2011;27:138-143.

35. Laupland KB, Zahar JR, Adrie C, et al. Severe hypothermia increases the risk for intensive care unit-acquired infection. Clin Infect Dis. 2012; 54(8):1064-1070.

36. Tiruvoipati R, Ong K, Gangopadhyay H, et al. Hypothermia predicts mortality in critically ill elderly patients with sepsis. BMC Geriatr. 2010;10:70.

37. Murio C, Soler X, Pérez M, et al. Acute exacerbation of chronic obstructive pulmonary disease in primary care setting in Spain: the EPOCAP study. Ther Adv Respir Dis. 2010;4(4):215-223.

38. Omachi TA, Blanc PD, Claman DM, et al. Disturbed sleep among COPD patients is longitudinally associated with mortality and adverse COPD outcomes. Sleep Med. 2012;13(5):476-483.
International Journal of COPD

\section{Publish your work in this journal}

The International Journal of COPD is an international, peer-reviewed journal of therapeutics and pharmacology focusing on concise rapid reporting of clinical studies and reviews in COPD. Special focus is given to the pathophysiological processes underlying the disease, intervention programs, patient focused education, and self management protocols.

\section{Dovepress}

This journal is indexed on PubMed Central, MedLine and CAS. The manuscript management system is completely online and includes a very quick and fair peer-review system, which is all easy to use. Visit http://www.dovepress.com/testimonials.php to read real quotes from published authors. 